\title{
Controlling processing in complex food systems: a potential route to food-allergy prevention
}

\author{
E.N. Clare Mills, ${ }^{\#, *}$ Neil M. Rigby ${ }^{\#}$, Ana I. Sancho, F. Javier Moreno ${ }^{\#}$ and \\ John A. Jenkins ${ }^{\#}$
}

The prevention of food allergy has two different dimensions: (1) the prevention of a reaction in an individual already sensitized and (2) the prevention of sensitization to food in the first place. The first of these is important as currently there is no proactive treatment for food allergy and consequently all that can currently be offered to individuals suffering from classical IgE-mediated food allergy is to avoid completely all problem foods coupled with emergency management of reactions for those with severe allergies. In addition to employing hazard-control procedures to minimize inadvertent contamination of foods with allergens, researchers and the food industry have been investigating options to reduce allergens in foods with a view to delivering allergic consumers with a wider choice of foods which are safe for them to eat. These can encompass a 'field-to-table' approach, involving elements of identifying raw materials and food naturally low in allergens, processing approaches to remove allergens and the development of 'null allergen' alternatives. The viability of these different approaches should be considered in the light of our current knowledge regarding the structures and properties of allergens in foods and illustrated with examples of allergens from fruits and vegetables, contrasting the properties of the PR10 proteins which are homologues of the major birch-pollen allergen Bet $\mathrm{v} 1$ and cause pollen-fruit/vegetable cross-reactive allergy syndromes, with those of the nonspecific lipid-transfer proteins (ns LTPs) which appear to be responsible for causing more severe allergies, including life-threatening reactions, in Southern Europe. This has been a major topic of the EU-funded project SAFE (Field-to-Table Strategies for Reducing Allergenic Risks Across Europe; SAFE-QLK1-CT-2000-01394), where the effect of the genetic background of apples, their maturation on the tree and subsequent post-harvest storage and processing on the levels of these different allergens has been studied. Preliminary results indicate that the different allergen types respond differently post-harvest and during physical food processing, including the thermal treatments employed during fruit-juice manufacture.

Physical food processing may both destroy and induce 'neo-epitopes' in food proteins and it maybe that the food matrix itself can affect the release and stability of allergens in foods, affecting their allergenic potential. This is an important consideration regarding the effect processing and the food matrix may have on promoting food allergies. Such factors may contribute to the potency of peanuts as allergenic foods, in contrast to the botanically related pea, which is rarely allergenic. The effect of both the inherent stability of the three-dimensional scaffold and the

\footnotetext{
\# Institute of Food Research, Norwich Laboratory, Norwich Research Park, Colney, Norwich NR4 7UA, UK

*Corresponding author: E-mail: clare.mills@bbsrc.ac.uk
} 
Chapter 4

matrix itself on stability of allergens digestion will be illustrated by important examples of plant seed storage-protein allergens belonging to the $2 \mathrm{~S}$ albumin and $7 \mathrm{~S}$ globulin families, drawing on the results of an EU-funded project ALLERGEST (The Effect of Gastrointestinal Digestion on the Allergenicity of Foods; QLK1-CT-200101239). 